\title{
STABILITY TESTS OF MODULE COIL (TOKI-MC) WOUND WITH AN ALUMINUM STABILIZED SUPERCONDUCTOR
}

\author{
T. Mito, M. Sakamoto, K. Takahata, N. Yanagi, A. Nishimura, S. Yamada, O. Motojima and J. Yamamoto
} National Institute for Fusion Science, Toki-shi, Gifu-ken, 509-52, Japan

Abstract--The Module coil (TOKI-MC) is a twisted solenoid coil wound with an aluminum stabilized superconductor as an R\&D program of Large Helical Device (LHD). The TOKI-MC can simulate the conductor and winding structure cooled by pool boiling helium, the twisted winding and the large electromagnetic force of the helical coils for LHD. The TOKI-MC was designed as a cryostable coil at an operating current of $20 \mathrm{kA}$, but the coil quenched around $17 \mathrm{kA}$ in excitation tests. The cause of quenches was thought to be the result of wire movement. Stability tests were also made and the measured recovery current $(13.6 \mathrm{kA})$ was less than the designed value $(23 \mathrm{kA}$ at $8 \mathrm{~T}$ ). The degradation of recovery current was due to the excess magnetoresistivity of the copper clad aluminum stabilizer. The stability of TOKI-MC was evaluated and compared with the data of short sample tests.

\section{INTRODUCTION}

The LHD is a fully superconducting heliotron type fusion experimental device. One of the main features of LHD is the use of superconducting coils for all the magnetic confinement coils (two helical coils and three pairs of poloidal coils). The helical coils are designed with pool boiling conductors considering the flexibility of the conductor for the three-dimensional helical winding.

The conductor of the helical coils is designed as a composite conductor with $\mathrm{NbTi} / \mathrm{Cu}$ superconductor and high purity aluminum stabilizer. Several cross-sectional patterns have been proposed for the conductor of helical coils. Some of them were made on an experimental basis and have been tested for superconducting property, stability [1] and mechanical property. The TOKI-MC is a twisted solenoid coil wound with one of the proposed conductors for the helical coils. The TOKI-MC can simulate the conductor and winding structure cooled by pool boiling helium, the twisted winding and the large electromagnetic force of the helical coils [2]. The excitation tests of TOKIMC also served as a long length sample test of the proposed conductor named Design-M.

\section{TOKI-MC COIL AND DESIGN-M CONDUCTOR}

The TOKI-MC was wound obliquely on an elliptical bobbin with the Design-M composite superconductor. The windings (17 turns $\times 14$ layers) are covered with thick stainless steel coil case simulating the cooling condition and the mechanical supporting structure of the helical coils. The inner radius, outer radius and width of the windings are about $0.4 \mathrm{~m}, 0.7 \mathrm{~m}$ and $0.4 \mathrm{~m}$, respectively. The central and maximum magnetic fields are $7 \mathrm{~T}$ and $7.6 \mathrm{~T}$ at the designed operating current of $20 \mathrm{kA}$. The coil covered with coil case was immersed in a liquid helium bath and

Manuscript received August 24, 1992 the windings were cooled by liquid helium penetrated into the cooling channel from holes at the bottom of the coil case. Each turn and layer of the windings were insulated with $3 \mathrm{~mm}$ thick GFRP spacers that covered $50 \%$ of the conductor surface and made cooling channels for liquid helium.

The cross-sectional view of Design-M conductor and its parameters are shown in Fig. 1 and Tab. 1. The conductor consists of a NbTi/Cu compacted strand cable at the center, four copper-clad aluminum stabilizers and a halfhard copper sheath. All elements were soldered together. The surface of the conductor was coated with $2 \mu \mathrm{m}$ thick copper oxide to enhance the heat transfer to liquid helium. In the TOKI-MC windings, the conductors were jointed with solder at each end of a layer. The maximum length of the conductor without joint was $160 \mathrm{~m}$ and the total length of the coil windings was $840 \mathrm{~m}$. The TOKI-MC was designed as a cryostable coil at an operating current of $20 \mathrm{kA}$. However the coil quenched around $17 \mathrm{kA}$ in excitation tests. The cause of quenches was thought to be the result of wire movement as described in a previous paper [3]. In this paper, the stability of the TOKI-MC coil is evaluated and compared with the data of short sample tests.

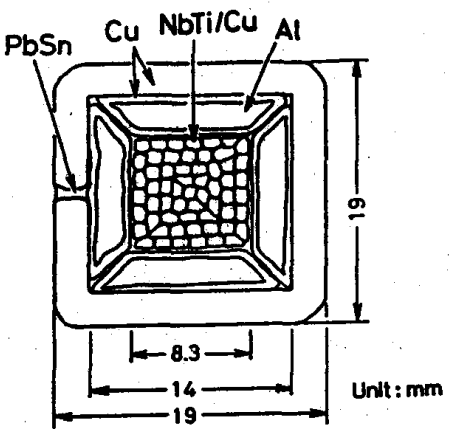

Fig.1. Cross-section of Design-M conductor

Table 1. Parameters of Design-M conductor

\begin{tabular}{|l|l|}
\hline Size & $19 \mathrm{~mm} \times 19 \mathrm{~mm}$ \\
\hline Nominal current & $20 \mathrm{kA}$ at $8 \mathrm{~T}, 4.2 \mathrm{~K}$ \\
\hline Critical current & $38 \mathrm{kA}$ at $8 \mathrm{~T}, 4.2 \mathrm{~K}$ \\
\hline Superconducting material & $\mathrm{NbTi}$ \\
\hline Number of strand & 58 \\
\hline Diameter of strand & $1.22 \mathrm{~mm}$ \\
\hline Cu/Sc Ratio & 1 \\
\hline Diameter of NbTi filament & $25 \mu \mathrm{m}$ \\
\hline Number of filaments & 1213 \\
\hline Twist pitch of filament & $17.0 \mathrm{~mm}$ \\
\hline Number of cable layers & 5 \\
\hline Twist pitch of cable & $127 \mathrm{~mm}$ for all layers \\
\hline Twist direction & Right for all layers \\
\hline Al cross section & $83 \mathrm{~mm}^{2}$ \\
\hline
\end{tabular}

$1051-8223 / 93 \$ 03.00 \odot 1993$ IEEE 


\section{STABILITY MEASUREMENT OF TOKI-MC}

Stability Margin

The stability margin of TOKI-MC was estimated by measuring the minimum heat input energy required to initiate a forced quench. A resistive heater of stainlesssteel foil (with a width of $20 \mathrm{~mm}$ and a resistance of 0.58 $\Omega$ ) was attached on the conductor surface at the 1st layer, 6 th turn of the winding. Voltage taps were located surrounding the heater as shown in Fig. 2. The angular position of the heater was selected to be 262 degrees (bottom of the coil) so that the conductors were aligned horizontally and the cooling condition might be the worst in the windings.
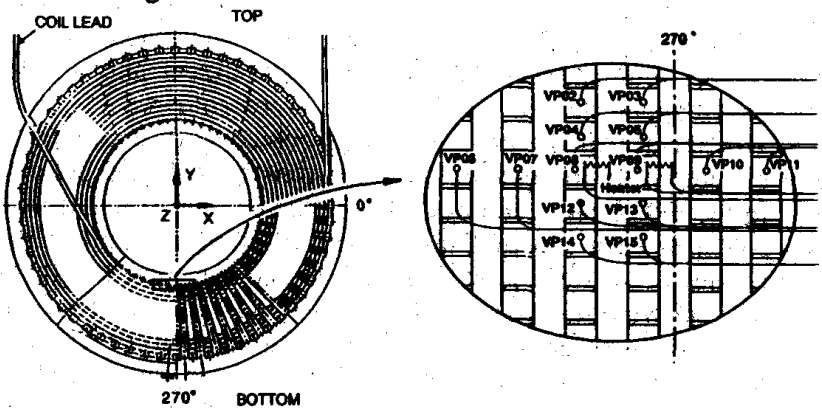

Fig. 2. Location of heater and voltage taps attached at 1st layer, 6th turn of TOKI-MC.

The plot of the minimum heater input energy required to initiate a quench versus the coil current are shown in Fig. 3 . The input signal of the heater was rectangular pulse with the time duration of $1 \mathrm{~s}$. Because of the long time duration of heater input, the heat input was dispersed by the heat transfer to liquid helium and the conduction along the conductor. Therefore this heater input energy can not directly mean the minimum quench energy. However, it can be said that the coil is very stable against the external heat input because of the extremely high thermal conductivity of the high purity aluminum.

\section{Recovery Current}

The recovery current was also measured using the same heater and voltage taps. At first, the normal zone was initiated by the heater at a current slightly higher than the recovery current. Then the coil current was decreased slowly to the recovery current at which the normal zone shrank. Figure 4a shows the current decay during the recovery current measurement with the balance voltage signal of the coil (the compensated coil voltage signal for flux change using a central voltage tap). The measured recovery current $(13.6 \mathrm{kA})$ was less than the designed value $(23 \mathrm{kA}$ at $8 \mathrm{~T})$. The designed value was calculated by Maddock's theorem [4] using the measured resistance of each component of the conductor and the measured heat transfer coefficient from copper oxide surface to liquid helium.

In Fig. $\mathbf{4 b}$, the resistivity of the conductor was measured at the same time using the short voltage taps near the heater. The conductor resistance stepped up from 0 to $1.0 \times 10^{-6} \Omega / m$ just after the heater input and kept almost constant value. This measured conductor resistance was three times larger than the designed value $\left(3.4 \times 10^{-7} \Omega / \mathrm{m}\right.$ at $6 \mathrm{~T})$. The conductor resistance was also measured during cool down just before the transition to superconducting state. The measured resistance was $1.1 \times 10^{-7} \Omega / \mathrm{m}$ that was almost the same as the designed value $0.93 \times 10^{-7} \Omega / \mathrm{m}$ at $0 \mathrm{~T}$. Therefore the increase of the resistance was not due to the deterioration of the residual resistance of pure aluminum

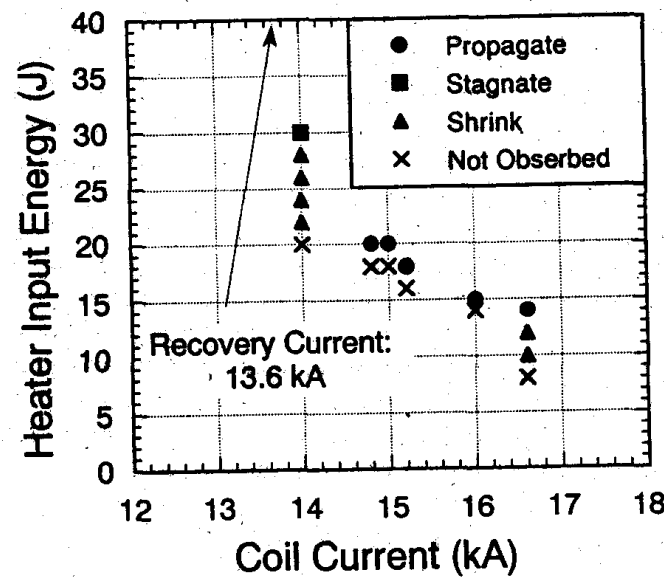

Fig. 3. Stability margin of TOKI-MC. Circle mark means normal zone propagates. Rectangle mark means normal zone stagnates keeping a constant voltage. Triangle mark means normal zone shrinks. Cross mark means normal zone is not observed.

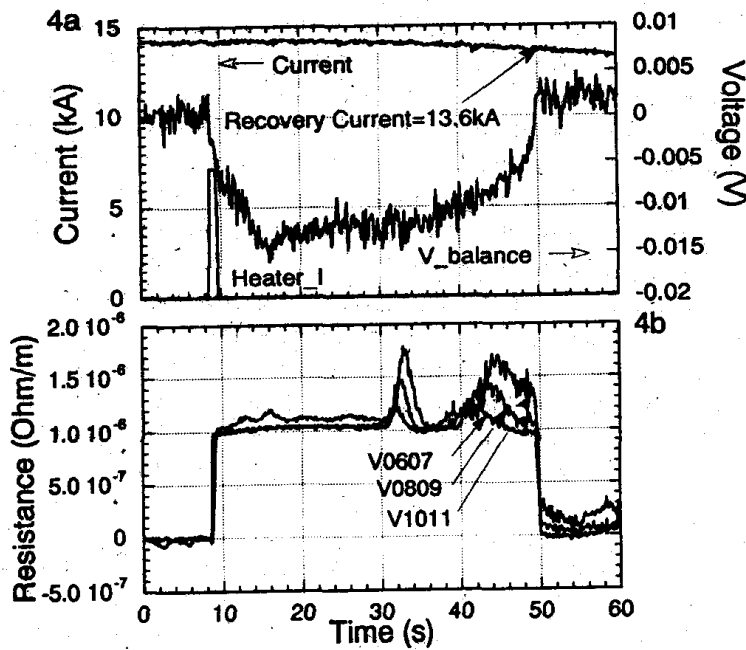

Fig. 4 Measurement of recovery current of TOKI-MC. 4a shows coil current, heater input and balanced voltage. $4 \mathrm{~b}$ shows conductor resistance measured by short voltage taps.

\section{COMPARISON WITH SHORT SAMPLE TESTS}

The reason of the degradation of the coil recovery current was examined as compared with the data of short sample tests. Two short sample conductors (the length of 
$1970 \mathrm{~mm}$ ) were arranged parallel with a gap of $3 \mathrm{~mm}$ and were inserted vertically into the split coil [5]. The conductors were soldered at the bottom (over a length of $400 \mathrm{~mm}$ ) to make a current loop. Figure 5 shows the recovery current of Design-M short sample conductor versus external magnetic field. The recovery current of the short sample was already lower than the designed value. Therefore the increase of the conductor resistance was not due to the mechanical stress from the large electromagnetic force in the coil

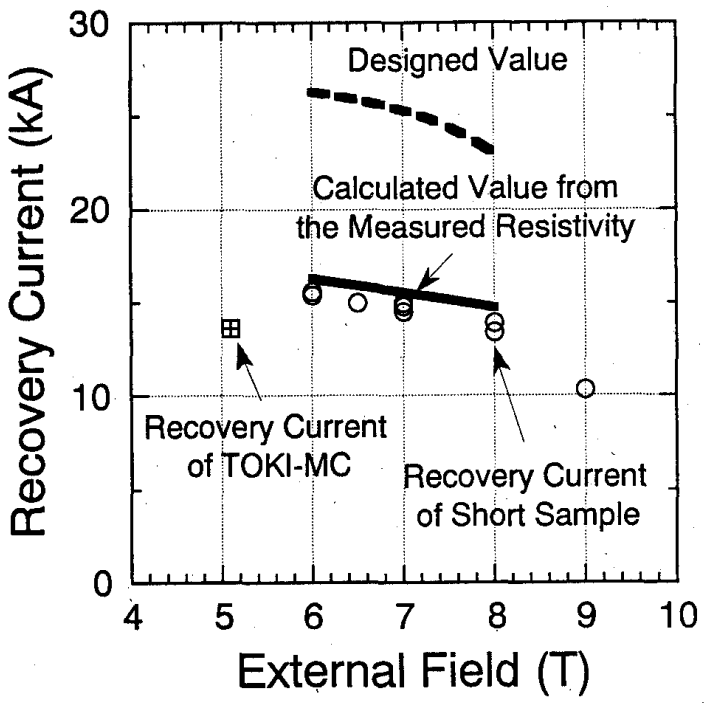

Fig. 5. Recovery current of Design-M vs. external field.

The resistance of short sample conductor versus the temperature with the external magnetic field as a parameter is plotted in Fig. 6. The temperature at the conductor surface was measured by $\mathrm{Au}(\mathrm{Fe})$-Chromel thermocouples compensating for the magnetic field effect. The resistance of the conductor was almost constant below $17 \mathrm{~K}$. The recovery current was measured below $17 \mathrm{~K}$ during the short sample tests. Therefore the increase of the resistance was not due to the temperature rise of pure aluminum. Figure 7 shows the calculation of the recovery current at $7 \mathrm{~T}$ by Maddock's equal-area theorem using the measured resistance curve of the conductor. The calculated result is plotted on Fig. 5 with a solid line that coincides well with the experimental values. In Fig. 7, the heat generation curve just came in contact with heat flux curve at $16.9 \mathrm{~K}$. It must be noticed that the rising rate of the heat generation versus the temperature was near the limit that Maddock's stability criterion could be applicable.

Figure 8 shows the conductor resistance of the short sample and the TOKI-MC coil versus the external magnetic field. The conductor resistance of TOKI-MC is a little higher than those of the short sample. This difference could be explained by the poorer cooling condition of the coil compared to the short sample. From Fig. 8, we estimated the temperature of the coil was more than $20 \mathrm{~K}$ as compared to the less than $17 \mathrm{~K}$ for the short sample. These decreases of the recovery current were also observed for the short sample test when the cooling condition became

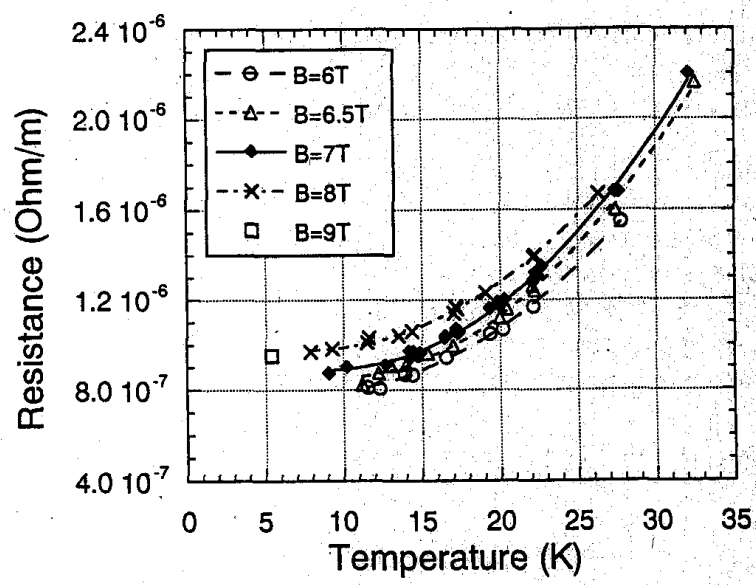

Fig. 6. Resistance of Design-M vs. conductor surface temperature.

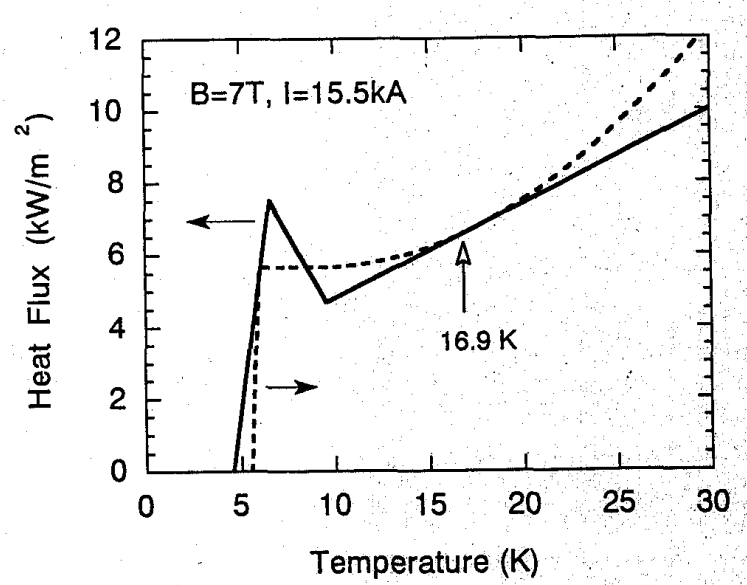

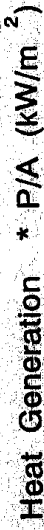

Fig. 7. Calculation of recovery current with Maddock's equal area condition at $B=7 \mathrm{~T}$ and $\mathrm{I}=15.5 \mathrm{kA}$.

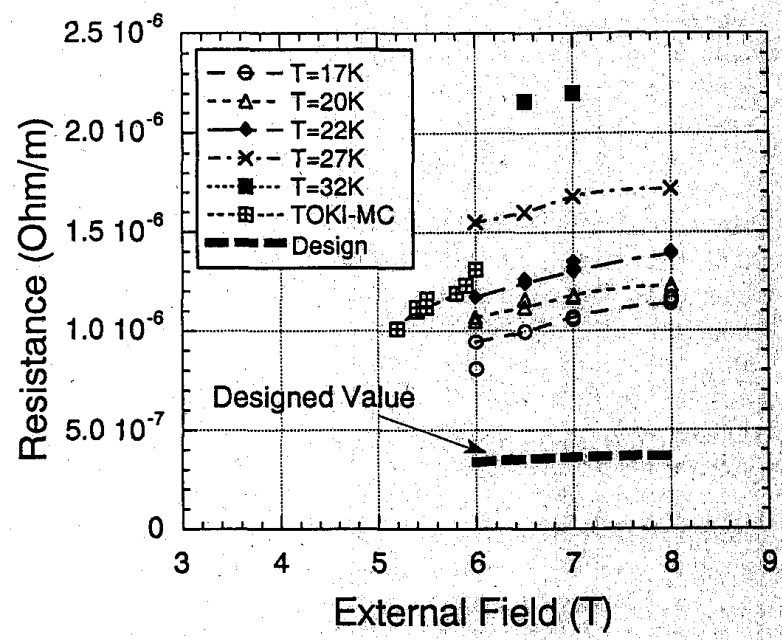

Fig. 8. Conductor resistance vs. magnetic field. 
worse. The short sample was covered with GFRP pipe to make $3 \mathrm{~mm}$ thick cooling channel. A heater named the bubble heater was mounted at the bottom of GFRP pipe to evaporate liquid helium and simulate vapor locking condition in the coil windings. As shown in Fig. 9, the recovery current decreased as the heat input to the bubble heater increased. Because of the difference of the direction of the cooling channel between the coil and the short sample, the cooling condition of the coil was much worse than that of the short sample.

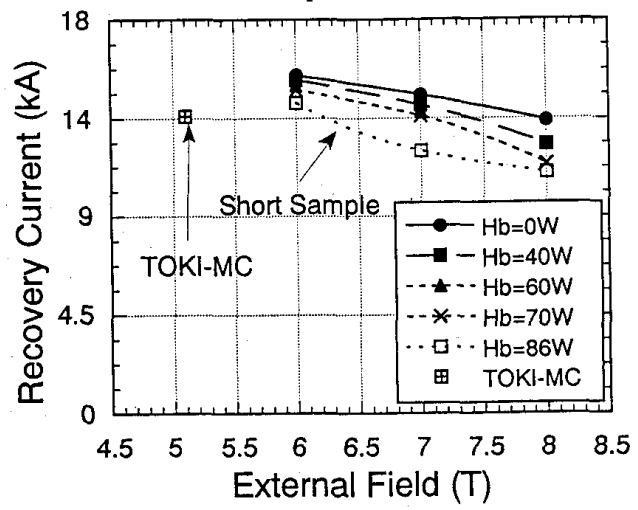

Fig. 9. Effect of cooling condition on the recovery current measured by short sample with bubble heater

\section{DISCUSSION}

The degradation of recovery current was caused by the increased resistance of the aluminum stabilized composite conductor. It was confirmed that the increase of the resistance was not due to defects of the materials. The effect of temperature rise and electromagnetic stress were also considered and were eliminated. It was reported by P.W. Eckels et al: that the magnetoresistivity of a pure aluminum composite clad with $\mathrm{Al}-\mathrm{Fe}-\mathrm{Ce}$ sheath becomes very large compared to a pure aluminum bulk without a sheath [6]. They explained that the mechanism of magnetoresistivity increase was related to Hall generation within the composite. Hall coefficients of aluminum and copper are different from each other in their polarity. $\mathrm{H}$. Kaneko et al. calculated the enhancement of magnetoresistance due to Hall current that may flow across the interface in aluminum-copper composite [7]. However, some experimental data can not be simply explained by the above Hall generation model. There is a possibility that Hall coefficients or other material parameters may change caused by the combination of materials with different Hall coefficients. The increase of magnetoresistance is an important singular phenomenon for aluminum stabilized composite conductor. We are investigating further the cause and the detail mechanism of the enhanced magnetoresistance.

As shown in Fig. 7, the heat generation of the aluminum composite conductor increased rapidly. The temperature gradient inside the conductor shifts the heat generation curve to low temperature region and may cause an instability and a temperature runaway of the conductor. Furthermore, the cooling condition of the coil is worse than that of the short sample. Therefore it is very important for stability of an aluminum stabilized composite conductor to minimize the temperature gradient inside the conductor.

The stability of the coil can not relay only on the stability of the conductor. The mechanical stiffness of the windings must be high enough to suppress the mechanical disturbance that can initiate a coil quench.

\section{CONCLUSION}

The TOKI-MC was designed as a cryostable coil whose recovery current was greater than the operating current From measurements of stability margin the coil was very stable against the external heat input because of the extremely high thermal conductivity of the high purity aluminum. The recovery current of the short sample conductor was, however, lower than the designed value caused by the excess of magnetoresistivity of a copper clad aluminum stabilizer.

The temperature dependence of the conductor resistance was measured and the resistance increased rapidly above $17 \mathrm{~K}$. Therefore it is very impotent for the stability of an aluminum stabilized composite conductor to keep the conductor at a low temperature and to minimize the temperature gradient inside the conductor when it becomes normal.. The cooling condition of the coil became worse than that of the short sample and some degradation of the stability was observed comparing to those of the short sample test. To make use of these results, we have been improving the conductor and the winding structure applicable to the construction of LHD.

\section{ACKNOWLEDGMENT}

The authors are grateful to many workers of Mitsubishi Electric Corporation, Sumitomo Electric Industries Ltd. for their contribution to the design and fabrication of TOKI-MC coil and Design-M conductor.

\section{REFERENCES}

[1] T. Mito, J. Yamamoto, K. Takahata, N. Yanagi, O. Motojima, "Development of superconducting conductors for Large Helical Device," IEEE Trans. on Magn., Vol. 27, pp. 2224-2227, March 1991.

[2] T. Mito et al., "Design and Fabrication of Module Coil as an R\&D Program for Large Helical Device," IEEE Trans. on Magn., Vol. 27, pp. 2361-2364, March 1991.

[3] M. Sakamoto et al., "Excitation Experiments of Module Coil (TOKI-MC) as an R\&D Program for Large Helical Device," presented at this conference, paper LLC-6.

[4] M.N. Wilson, SUPERCONDUCTING MAGNETS, New York: Oxford University Press, 1983, pp.91-129.

[5] T. Mito et al., "Short Sample Tests of Full-Scale Superconducting Conductors for Large Helical Device," IEEE Trans. on Magn., Vol. 28, pp. 214-217, January 1992.

[6] P.W. Eckels, N.C. Iyer, A.T. Male, J.H. Parker, Jr and J.W. Coltman, "Magnetoresistance: the Hall Effect in Composite Aluminum Cryoconductors," Cryogenics, Vol. 29, pp. 748-752, July 1989.

[7] H. Kaneko, N. Yanagi, "Enhancement of Magnetoresistance due to Hall Current in AluminumCopper Composite," Cryogenics to be published. 